\title{
Auditoria Forense
}

\author{
Guillermo Williams Bautista ${ }^{1}$
}

\section{Introducción:}

A menudo cuando platicamos con algunas personas y les explicamos que nos dedicamos a realizar auditoría forense, vemos en sus ojos algún sentido de sorpresa e incertidumbre, pero, esa reacción ya es conocida, por lo tanto aclaramos, no, nos dedicamos a abrir muertos, y en realidad esperamos nunca hacer algo como eso.

De hecho, nos dedicamos a la prevención, detección y disuasión de fraude en las empresas, así que seguramente usted está pensando, ¿pero cómo se nos ocurrió especializarnos en auditoría forense?

Bueno, entre las razones personales existe la experiencia de que habiéndonos dedicado a la auditoría financiera, y al platicar algunos consejos de administración, los dueños o los tenedores de acciones, comentaban constantemente, está bien, dicen que nos faltan activos, que nuestras cuentas bancarias deberían de tener más dinero, que nuestros pasivos no fueron registrados, que nuestros estados de resultados integrales no reflejan la actividad de nuestra empresa, que debería tener más utilidades y que por lo tanto sería recomendable que analizáramos en otro contexto nuestra información económica, pero... ¿porque no nos dicen cuál es la solución?

A lo largo de este artículo intentaremos detallar algunas de las actividades más importantes que se realizan durante el desarrollo de una auditoría forense y usted mismo podrá determinar si este tipo de auditoría podría ser una respuesta a la incógnita planteada,

\section{Desarrollo}

¿De dónde proviene el término forense?

Es de amplio conocimiento (no se atribuye a ningún autor) que el término "forense" proviene del latín "forensis" que significa "público y manifiesto" o "perteneciente al foro"; a su vez, "forensis" se deriva de "forum", que significa "foro", "plaza pública", "plaza de mercado" o "lugar al aire libre".

También se dice que antiguamente en Roma y en las otras ciudades del Imperio Romano algunas de las actividades principales como las asambleas públicas, las transacciones comerciales y las actividades políticas se realizaban en la plaza principal. En dichos foros (plazas) también se trataban los negocios públicos y se celebraban los juicios de manera abierta, o a la luz pública; por eso, cuando una profesión sirve de soporte, asesoría o apoyo a la justicia para que se juzgue el cometimiento de un delito, o que ayuda a sacar a la luz pública la verdad sobre determinado hecho, se le denomina forense, de allí el término de auditoría forense.

\section{Concepto}

"La Auditoría Forense es una rama de la auditoría que se orienta a participar y coadyuvar en la investigación de fraudes, es decir de actos conscientes y voluntarios con los cuales se burlan o eluden las normas legales, o se usurpa lo que por derecho corresponde a otros sujetos"1, mediante el uso de mecanismos dolosos para obtener una ventaja o un beneficio ilícito.

Lógico es pues que este tipo de auditoría requiera ciertas habilidades especiales en algunos temas centrales como son los que se enumeran a continuación:
0

${ }^{1}$ Contador Público Certificado. M.C.I.E., C.R.M.A., C.F.E. Ex presidente del Colegio de Contadores Públicos de Sonora. gwilliamsb@live.com.mx 


\section{Esquemas de Fraude y Transacciones Financieras:}

He aquí donde los contadores públicos tienen cierta ventaja en el trabajo de Auditoría Forense dado que el conocimiento de los conceptos básicos de contabilidad (Normas de Información Financiera) les brinda una visualización idónea no solo de cómo deben de registrarse las transacciones financieras en la empresa, sino además de la forma en que deben de analizarse sus resultados, transitar las operaciones financieras a través del sistema informático y visualizar las actividades operacionales que debieron intervenir en su formulación.

1 Diccionario Jurídico Guillermo Cabanellas, historiador, abogado, editor y escritor, destacado laboralista en Iberoamérica, Argentina y Paraguay

Esto resulta imprescindible para delimitar si existen evidencias de la existencia de fraude en los Estados Financieros, apropiación indebida de activos a través de la recepción de efectivo en los diversos procesos en los que este rubro participa como cobranza de clientes, ventas en efectivo, reembolso de gastos en caja chica, etc. además de otro tipo de esquemas como reembolsos de viáticos, apropiación indebida de inventarios, la corrupción y el soborno, el robo de propiedad intelectual, el fraude a través de y en instituciones financieras, los fraudes utilizando tarjetas de crédito, cheques y transferencias bancarias, el fraude en instituciones de salud y las correlacionadas en seguros y fianzas, el fraude de los mismos consumidores, y el correlativo a través de esquemas de contratos y procuración, solo por mencionar algunos.

\section{Leyes}

Por otra parte es sumamente importante que el auditor forense conozca las bases legales sobre las cuales se desarrollan las actividades en nuestro país e inclusive, para los que ostentan una certificación mundial, como se manejan las leyes a través de los distintos países en donde se celebran operaciones a un nivel global como lo hacen actualmente gran parte de las empresas, mismos conocimientos que se utilizaran para:

Visualizar correctamente el sistema legal al que está constreñida la empresa para así delimitar los conceptos legales que le aplican y que van a trastocar la esfera jurídica del fraude en particular que se esté tratando o abordando como puede ser por ejemplo el esquema legal específico que se debe de tratar en caso de que la empresa se haya declarado en bancarrota, en concurso mercantil o el que sea aplicado en el país donde residan principalmente las operaciones de la empresa que se trate así como las repercusiones naturales que tendrá en el nuestro y las formas de reporte que para lo mismo se deben de realizar cumpliendo con las formas que refieren el marco normativo de los dos países, o como pudieran ser por otro lado todas las disposiciones aplicables y que son necesarias para cumplir con las leyes anti-lavado.

Pero algo sin duda alguna crucial, es toda la esfera legal que se debe de respetar para realizar y proteger los derechos individuales dentro de la investigación forense y en los diversos ámbitos donde se realiza (las leyes pueden otorgar premisas y prerrogativas diferentes en el sector público o privado, en el ámbito civil, administrativo, mercantil, fiscal o penal por ejemplo), por lo tanto hay que conocer el contexto criminal o civil, respetando los principios básicos de la custodia de la evidencia, preparándose para ser un testigo experto o para realizar una pericial en alguna materia como la contable, pero que bien pudiera ser en materia de 


\section{Reportes de investigación}

sistemas asimismo para preparar al equipo forense para una cuestión de ingeniería, grafoscopio, documentos copia, minería de datos, etc., entender diferencias entre sistemas legales inquisitorios o adversarios, examen directo o cruzado, entre otras circunstancias sumamente importantes en la participación del auditor forense.

\section{Investigación}

Otra de las materias importantes es desarrollar cualidades específicas de investigación como planear y conducir una investigación de fraude, desarrollar la metodología específica del examen de fraude que se trate, desarrollando un plan de respuesta, como pudiera ser el conservar datos importantísimos de confidencialidad, elaborar teorías de fraude, elaborar y verificar perfiles de los defraudadores, obtener la evidencia documental, examinar apropiadamente la misma, consultar en el momento preciso a los expertos que nos puedan ayudar a obtener información valiosa, saber examinar huellas en los diferentes instrumentos inmiscuidos en la información, saber cómo extraer información vital de las computadoras sin contaminar la cadena de custodia, desarrollar cualidades como entrevistador tanto en la preparación de la entrevista como en el desarrollo sin perder nunca de vista las consideraciones legales para efectuar la entrevista, considerando los elementos de comunicación que se dan de forma en una conversación, tomando en cuenta los inhibidores que no permiten que fluya dicha comunicación, facilitando la misma y propiciando una sinergia con habilidades cognitivas, tomando en cuenta la tipología de los cuestionamientos, preparando las preguntas de apertura, las de información trascendente, las de cierre y las de aseguramiento, pero definitivamente a lo que se le puede llamar todo un arte es al hecho de obtener declaraciones de los sospechosos o posibles implicados en el fraude, pero sobre todo, al particular de obtener declaraciones firmadas en donde se admita la culpa del hecho sobre el cual se desarrolla la auditoría forense, y todo esto, por
Todo esto sin considerar aún que en ocasiones es importantísimo acudir a operaciones encubiertas, si, de esas que usted ve en los programas de televisión, y desde luego considerándose todas las fuentes de información ya sea públicas o no públicas, como son el registro público de la propiedad (pública) u otras que aun no siendo propiamente de esta naturaleza cuentan con una serie de limitaciones que el caso implica pero que pueden servir en la investigación (registros en Facebook por ejemplo), además en esta fase de investigación se cuenta con una parte importantísima que es el análisis de datos y las herramientas de reporte, para lo cual se utiliza lo que se conoce como auditoría a tecnologías de la información.

También es necesario el trazar las operaciones ilícitas a fin de obtener todo lo referente al caso que se está investigando, utilizando métodos directos e indirectos con el propósito de trazar precisamente la información financiera que pueda estar dispersa y que casi siempre resulta de naturaleza concluyente para nuestra investigación forense pasando así a los reportes escritos de manera apropiada, organizando la información, estructurando el reporte y facilitando la comprensión de los lectores principales.

Por lo tanto, es sumamente importante llevar un check list de todo lo que refiere al proceso de investigación ante el reto que comprende el desarrollo y conclusión de la etapa. 


\section{Disuasión y prevención de fraude}

Sin duda alguna, la fase de del presente trabajo que cobra más valor es precisamente la disuasión y prevención de fraude, en la vida profesional personalmente le he denominado colocación de banderas antifraude, que consiste en el estudio, fortalecimiento y desarrollo de control interno, el bastión más importante desde mi punto de vista para lograr el éxito operacional y financiero en una empresa.

Para lo cual, también es necesario entender el comportamiento criminal, por ejemplo cuales son los factores que pueden hacer "estallar" la bomba de tiempo que le presenta la oportunidad de cometer un ilícito a una persona proclive al fraude, cuales son las características, por ejemplo, de un delincuente de "cuello blanco" y como se conforma el crimen organizacional y el crimen ocupacional.

Por lo tanto, siempre es recomendable acudir a las mejores prácticas dictadas por la OCDE en cuestión de "gobierno corporativo" que no es otra cosa más que mejorar el ambiente de control o la forma en que el "tone at the top" o el tono en que los máximos niveles de jerarquía de la organización trabajan para la toma de decisiones de la empresa.

Además hay que delimitar y entender muy bien cuál es la responsabilidad de la gerencia en torno al fraude, la cual si bien es cierto que no debe ser operadora de todo en la empresa, si es responsable principalmente de establecer claramente los objetivos, administrar y asegurar los riesgos de la empresa, lo cual refiere evaluarlos, establecer tolerancia a los mismos, darles respuesta, fomentar una comunicación apropiada a través de la estructura de la empresa, monitorear y establecer controles específicos, supervisar y retroalimentar con las deficiencias encontradas al sistema, asimismo, es responsable por generar una cultura de cumplimiento y ética, determinar políticas de resguardo documental apropiadas, y en general practicar una cultura de responsabilidad ante la detección de fraude, administrar programas de prevención, vigilar los posibles conflictos de de intereses, elaborar políticas antifraude apoyándose todo esto de manera preferente en esquemas como CO$\mathrm{SO}^{\odot}$, COSO $\mathrm{ERM}^{\odot}$; COSO interrelación de componentes $^{\odot}$ (Coso ${ }^{\odot}$ es The Committee of Sponsoring Organizations of the Treadway Commission (COSO) , COMITÉ DE ORGANIZACIONES DEL TRATADO), además de las normas ISO 31000, ISO 31000 e ISO 31004.

Todo esto le va a permitir a usted tener una visión diferente de la empresa y trabajar, precisamente en sentido preventivo.

\section{Habilidades y competencias del Auditor}

\section{Forense}

Por lo tanto, el auditor forense debe en todo momento, a través de sí mismo y de su equipo especializado, durante la realización de diferentes esquemas de trabajo, lograr desarrollar y obtener mediante habilidades y competencias las siguientes cualidades entre otras tantas:

$\Rightarrow$ Conocimiento del negocio, comprensión de la forma en que este opera, identificación de los planes estratégicos que se desarrollan (aun no estando formalizada esta), la forma en que se lleva el control (Algunos contadores en México cuentas con una visión limitada de esto ya que solo se ubican en los controles específicos los cuales son solo una parte del control interno de la empresa) y por supuesto observar como fluye la comunicación formal e informal tanto dentro de la empresa como hacia afuera de la misma, a nivel horizontal, vertical y diagonal -la comunicación es una parte fundamental del control interno- etc.; como se puede observar, debe conocer de la forma más completa posible a la organización.

$\Rightarrow$ Adoptar una mente escéptica, estratégica, capaz de elaborar teorías de acuerdo con métodos científicos a fin de cumplir con una función investigadora de la gestión empresarial. 


\section{Reportes de investigación}

$\Rightarrow$ Adoptar una mente escéptica, estratégica, capaz de elaborar teorías de acuerdo con métodos científicos a fin de cumplir con una función investigadora de la gestión empresarial.

$\Rightarrow$ Estar a la vanguardia en el conocimiento actual y del desarrollo de tecnologías de información, el defraudador en muchas de las ocasiones es experto en ellas.

$\Rightarrow$ Mantener la mente abierta, desarrollando e innovando técnicas, procedimientos y métodos de auditoría para prevenir, detectar, disuadir y luchar contra el fraude de manera efectiva, hay que recordar que el defraudador va un paso delante de cualquiera dentro y fuera de la organización en la cual va a desarrollar sus trabajos, además es experto en innovar y encontrar debilidades en el sistema de control interno, de ninguna manera puede ser subestimado.

$\Rightarrow$ Mantenerse continuamente motivado, detectar un fraude no es cosa fácil cuando no se han encontrado previamente indicios de ello, el trabajo del defraudador es esencialmente tapar las huellas de su actuar delictivo, ha ocupado tiempo y esfuerzos importantes planeando como no ser descubierto.

$\Rightarrow$ Desarrollar cada vez mejores habilidades de investigación, el fraude ocurre en cualquier tiempo, en cualquier área, bajo cualquier forma, puede ser registrado en Estados Financieros, puede ocultarse tras ellos e inclusive puede estar fuera de los mismos.

$\Rightarrow$ Etc.

El auditor forense debe tener habilidad y destreza a través de sí mismo o de su equipo de trabajo para el desarrollo de actividades en cuestiones tales como:
- Debe ser un perfecto analista de información financiera, realizando análisis horizontal, vertical, a nivel comparativo entre cifras de estados financieros y no financieros a medida de saber dar "tiros de precisión".

- Debe de conocer todas las técnicas de investigación posibles, en todos los ámbitos -legales, tecnológicos, de campo, de entrevista, de correlación de datos, científicos, etc.-, e inclusive contemplar nuevas formas que no se han explorado por otros colegas ya que ningún fraude es igual a otro.

- Tener plena conciencia del manejo de la evidencia, establecer una cadena de custodia, encontrar cual consiste en una evidencia circunstancial y una plena, saber concatenar las mismas, etc.

- Conocer el marco de control de cada empresa y el ideal que se utiliza en cada campo de actuación, ejemplo: seguimiento de procedimientos legales, sus plazos y sus consecuencias, en el caso de información tecnológica manejo de marcos de control como COBIT, ITIL, ISO 27000, ISO 27001.

- Perfecto conocimiento del manejo y administración de riesgos, integrando mapas de riesgos, mapas de calor, planeación estratégica de respuesta a los mismos, etc.

- Capacidad de manejo de equipos multidisciplinarios, entendiendo los planes de trabajo, el manejo de estrategias y las conclusiones de diferentes tipos de profesionistas como los psicólogos al elaborar perfiles de defraudadores, solo por citar un ejemplo.

El fraude en frase:

"El humo como recurso de una estafa nubla primero el entendimiento y después nuestra capacidad de ver lo que realmente es" ${ }^{2}$

${ }^{2}$ Revista especializada en fraude de la ACFE, Capítulo México. 


\section{Informes de resultados y manejo oportuno de hallazgos}

Durante el trabajo de auditoría forense, el auditor debe tener un "feeling" apropiado para presentar de manera oportuna aquellos hallazgos que presentan un riesgo, cuando, de seguir ocurriendo puedan lesionar de forma importante a la empresa, esto, aún y cuando no sea tiempo de presentar el informe final, situación que se torna sumamente delicada, ya que un manejo de forma inoportuna o inapropiada, puede advertir a los defraudadores sobre el hecho de que están siendo observados, pudiendo proceder los mismos a contaminar los escenarios en que se está cometiendo el delito, por lo tanto, este punto es de los más importantes en la comunicación con los "stakeholders" o grupos de interés.

Además, en la presentación de los resultados se debe de tener una cautela profunda en la redacción a fin de no ser sujetos o verse inmiscuidos en denuncias por calumnias, injurias, falso aprisionamiento, obtención ilegal de pruebas, etc., además, recuerde usted que el único en resolver si alguien resulta ser un defraudador o no, es el juez que está resolviendo en la instancia que se trate.

También es importante el reconocer cuando el trabajo de auditoría forense no ha llegado a los resultados esperados por las demás personas, jamás se puede prometer formalmente que se va a encontrar un fraude, ni puede hacer creer que lo va a realizar, a lo más que el auditor forense deberá a llegar es a encontrar los indicios suficientes para que alguien pueda ser demandado por fraude en la esfera correspondiente.

Producto del ejercicio profesional, el auditor forense adquiere destrezas como las siguientes:

- Agudeza, escepticismo, análisis crítico, integridad.

- Profundidad en el análisis, generación de dudas razonables y sospechas, búsqueda de indicios.
- Visualización de riesgos.

- Observar las excepciones y explorar en la "parte oscura" de la información.

- Es capaz de apoyarse y elaborar métodos científicos

\section{Creación de equipos multidisciplinarios}

Claro está que para lograr todo esto es necesario que se conjunte una serie de profesionales con los conocimientos necesarios y que se destaquen por las competencias particulares que se requieren para una auditoría de este tipo, en la vida profesional nos hemos encontrado con personas sumamente valiosas en el Estado de Sonora y que demuestran un perfecto conocimiento de las materias necesarias, las cuales cito a efecto de mostrar que en Sonora hay personas de mucha valía y conocedoras del tema, además de ver la manera en que en ocasiones se conforma este tipo de equipos multidisciplinarios:

Licenciado en Derecho: René Alejandro Rojas Corral, miembro de ACFE $^{3}$

Licenciada en Derecho: Alejandra Burruel Villanueva, miembro de ACFE $^{3}$

Licenciado en Informática: Héctor Fernando Bustamante Federico, miembro de $\mathrm{ACFE}^{3}$

Ingeniera en Sistemas: Hortensia Monge Miranda, miembro de $\mathrm{ACFE}^{3}$

Psicólogo: Carlos Enrique Villagrana Borjòn, reconocido a nivel internacional por sus diversos trabajos de investigación.

Contador Público: Alberto Castañeda Rosas, miembro de $\mathrm{ACFE}^{3}$

${ }^{3}$ ACFE es la Asociación de Examinadores de Fraude Certificados con presencia mundial, es la organización especializada en Auditoría Forense. 


\section{Reportes de investigación}

\section{Enfoque de distintas auditorías}

A efectos de establecer algunas diferencias entre algunos tipos de auditorías y la auditoría forense, se presenta un recuadro con algunas adecuaciones propias, que fue citado en material editado por la auditoría superior de la federación el cual se titula "introducción a la auditoría forense" desarrollado por la Unidad General de Administración, y el Instituto de Capacitación y Desarrollo en Fiscalización Superior.

Diferencias y similitudes entre Auditoría Forense, Financiera e Interna.

\section{Auditoría Forense}

\section{Importancia}

Necesaria para investigar y prevenir ilícitos relacionados con el fraude

\section{Propósito}

Prevenir, disuadir e investigar presuntos actos de fraude, colocar banderas anti-fraude

Alcance

Periodo que cubre el delito, Periodos definidos, desde su inicio hasta la generalmente de un año finalización, instauración legales

\section{Orientación}

Retrospectiva

en incluyendo de acciones

\section{Auditoría Interna}

Necesaria para lograr y mantener un buen sistema financiero
Auditoría Financiera

Formular y expresar opinión sobre razonabilidad de estados financieros
Necesaria para el monitoreo de los controles y procesos internos de la entidad

investigación y con proyección a fortalecer controles.

la A la situación financiera, resultados, cambios en e patrimonio y flujo de efectivo
Periodos definidos generalmente de un año 
No existen normas plenamente definidas dada la complejidad e innovación de las formas de operar por parte de los defraudadores. Generalmente se recurre a la investigación la cual puede verter diversas técnicas y métodos científicos de medición dependiendo del caso.
Verificación de la aplicación de las Normas de Información Financiera por las empresas emitidas en México por el CINIF, a través de las Normas Internacionales de Auditoría, emitidas para México y Latinoamérica por el IMCP
Normas Internacionales para el

Ejercicio Profesional de la Auditoria Interna, emitidas por el IIA

\section{Técnicas}

Parte de las técnicas y procedimientos Se basa en las técnicas de de la auditoría y de las que se Auditoria

desarrollen en la investigación.

Evaluación de la Estructura del Control Interno

Indispensable

\section{Entrevistas}

\begin{tabular}{|c|c|c|}
\hline $\begin{array}{l}\text { Se orientan a los denunciantes, } \\
\text { informantes, testigos e investigados. }\end{array}$ & $\begin{array}{l}\text { Esporádicas, con enfoque al } \\
\text { área auditada. }\end{array}$ & $\begin{array}{l}\text { Necesarias, con enfoque al área } \\
\text { auditada. }\end{array}$ \\
\hline
\end{tabular}

Depende del caso específico

objeto de la investigación.

\section{Personal Ejecutor}

\begin{tabular}{l|l|l} 
Multidisciplinario y con & Contadores públicos & Multidisciplinario \\
participación interinstitucional & & \\
\hline Informe de Auditoria &
\end{tabular}

\section{El contenido del informe varía según}

la naturaleza y caracteristicas

del caso investigado,

generalmente se emite informe corto e informe largo, aun así nunca puede perderse de vista que muy probablemente el caso termine en proceso jurídico, por lo que pueden ser necesarias periciales profesionales.

\section{Conclusiones de Auditoria}

Pueden resultar diversas, la auditoria desde un inicio debe de ser preparada para en su caso, acudir a juicio por la vía civil, mercantil, fiscal, penal, administrativa, etc. dependiendo de los resultados que deben ser claramente detallados en el informe final en el cual se formulan los posibles indicios de la infracción en particular.

Frecuencia

En cualquier momento

Generalmente al año la máxima autoridad. Inciden sobre conclusión principal.
Es necesario, generalmente solo se evalúan los controles especificos y no el control interno de la organización.

Se basa en las técnicas de Auditoría Interna

Indispensable

Auditores, contadores públicos

auditor sobre los estados financieros $y$ sus anexos, en ocasiones, el informe de cumplimiento tributario y la comunicación a contenido del informe son acerca de la funcionalidad de la gestión de la entidad, controles y operaciones asi como de las incidencias detectadas asuntos financieros. El dictamen es la

Generalmente al ano
principal

Fuente: Elaboración propia. 


\section{Reportes de investigación}

\section{Decálogo profesional del auditor forense y del examinador de fraudes}

A continuación se expone un conjunto de normas o consejos profesionales para los auditores forenses, presentado por la Dra. Muna Dora Buchahin Abulhosn en la publicación de la "Revista Técnica sobre Rendición de Cuentas y Fiscalización Superior No. 8". El propósito de este Decálogo es promover que los auditores cuenten con las habilidades y aptitudes (profesionalismo, experticia, mentalidad investigadora y analítica, oratoria, argumentación, mediación, negociación, y creatividad) adecuadas en materia de prevención, detección e investigación de fraudes y otros ilícitos.

I. Mantenerse actualizado en su ámbito de especialidad. Los expertos están obligados a poseer todo el conocimiento posible sobre su materia; a mantenerse al día a través de la consulta periódica de publicaciones científicas; la asistencia a congresos, cursos, seminarios, talleres, y el constante intercambio de experiencias y opiniones.

II. Fomentar el pensamiento crítico y una efectiva comunicación oral y escrita.

III. Desarrollar su labor con independencia, imparcialidad y prudencia. Los expertos desempeñarán su labor de manera objetiva, sin deformar ni tergiversar el objeto de estudio; evitarán ceder a consideraciones personales o a otro tipo de intereses, y desarrollarán en todo momento su actividad con el debido cuidado y entrega profesional y desarrollarán en todo momento su actividad con el debido cuidado y entrega profesional.
IV. Utilizar métodos y técnicas de investigación científicas adecuadas en la búsqueda de la verdad. Los auditores -investigadores deberán adoptar una actitud analítica que sólo admita conclusiones basadas en hechos verificables. En este sentido, los expertos deberán proceder con todo rigor científico al establecer la metodología y técnicas particulares que aplicarán para tal fin.

V. Ser claro y preciso en la redacción y conclusiones de su informe y dictamen. Cuando los peritos acudan en calidad de testigos expertos, deberán explicar sus hallazgos en forma clara y escrupulosa, así como trasmitir su conocimiento profesional con firmeza.

VI. Emitir su dictamen sobre cuestiones técnicas y científicas, reservándose sus opiniones subjetivas, o sin sustento legal. Los peritos forenses son profesionales con preparación técnica y científica, por lo tanto pueden testificar en juicios y expresar su opinión de experto en los dictámenes, pero deben de reservarse su opinión o alegación sobre la presunta culpabilidad o inocencia del imputado, pues no hay que olvidar que su rol no es el de juzgador.

VII. Fundar sus conclusiones en hechos verificables. Los expertos deberán corroborar empíricamente sus enunciados a través de la observación y la experimentación, para lo cual podrán hacer uso de una amplia variedad de técnicas derivadas de la investigación científica. 
VIII. Ser conscientes de las limitaciones de su capacidad científica. Los expertos deben de ser conscientes de lo que saben y de lo que ignoran. Cuando éstos se enfrenten a problemas de alta especialidad es necesario que cuenten con la experiencia y los conocimientos necesarios para resolverlos; y en su caso, allegarse de toda la información útil que les permitan tomar decisiones adecuadas.

IX. Coadyuvar eficazmente con las autoridades en el esclarecimiento de los hechos. Los auditores investigadores han de respetar y amar, ante todo y sobre todo, la verdad y la justicia

$X$. Excusarse de dictaminar sólo por razones técnicas o legales; esto significa que, cuando los expertos se enfrenten a un problema cuya solución no está al alcance de sus conocimientos o posibilidades de acuerdo con su campo de especialidad, deberán excusarse de emitir un dictamen. Lo mismo ocurre para el caso de existir conflicto de intereses o algún impedimento para que dictaminen con la estricta objetividad e imparcialidad necesarias.

XI. Tener mente abierta ante las objeciones metodológicas y técnicas que cuestionen sus dictámenes. En el marco del sistema penal acusatorio, los expertos deberán estar preparados y tener una actitud serena e inteligente para defender sus dictámenes ante cualquier crítica que se formule en la etapa de juicio oral, pues en virtud del principio rector de contradicción, las partes podrán conocer, controvertir o confrontar los medios de prueba, así como oponerse a las peticiones y alegatos de la otra parte.

XII. Argumentar y opinar con datos incontrovertibles, preguntar para aclarar, no distraer su mente ni atención durante su trabajo.

XIII. Mantener un compromiso permanente con la legalidad. Cualquier mínima sugerencia de desviación respecto a la ética profesional deberá encontrar en los expertos la más rotunda negativa.

XIV. La personalidad forma parte esencial en cualquier proceso. El manejo del lenguaje verbal y no verbal es parte fundamental de la actuación de los peritos expertos. Éstos debe ser pulcros, claros y asertivos.

$\mathrm{XV}$. Nunca improvisar.

\section{Conclusión}

La auditoría forense es una actividad que requiere de muchas habilidades, competencias, capacidades y destrezas por lo que prácticamente es imposible realizarla sin el apoyo de un equipo multidisciplinario con conocimientos desarrollados al respecto ya que también debe de efectuarse con mucha responsabilidad; Este tipo de auditorías en la actualidad resulta indispensable para el desarrollo pleno de las empresas, después de todo, el fraude es el cáncer de las empresas y los agentes que los provocan, es decir los defraudadores, son personas con un perfil tal, que son capaces de engañar a la mayoría de las personas, trabajando en un entorno que conocen a la perfección y diseñando sistemas de encubrimiento de los ilícitos, por lo tanto, a diferencia plena de los fraudes que se descubren por accidente, el dedicarse profesionalmente a "sacarlos a la luz pública", constituye un reto apasionante para cualquier profesionista, incluidos los contadores públicos quienes ostentan una ventaja plena en la actividad ya que conocen de manera idónea los procedimientos y técnicas de auditoría, el análisis de información financiera, la manera en que debe transitar la información a través del sistema contable, aún sin embargo debe de apoyarse en un equipo brillante por su manera de trabajar en el contexto que a cada quien le corresponde.

\section{Bibliografía recomendada}

${ }^{1}$ Diccionario Jurídico Guillermo Cabanellas, historiador, abogado, editor y escritor, destacado laboralista en lberoamérica,

Argentina y Paraguay

${ }^{2}$ Revista especializada en fraude de la ACFE, Capítulo México.

${ }^{3}$ ACFE es la Asociación de Examinadores de Fraude Certificados con presencia mundial, es la organización especializada en Auditoría Forense. 